\title{
Principais estratégias usadas para promoção à saúde dos profissionais de enfermagem na pandemia da Covid-19
}

\author{
Main strategies used to promote the health of nursing professionals in the Covid-19 pandemic \\ Principales estrategias utilizadas para promover la salud de los profesionales de enfermería en la \\ pandemia Covid-19
}

Recebido: 26/08/2021 | Revisado: 03/09/2021 | Aceito: 15/09/2021 | Publicado: 16/09/2021

\author{
Shirley Vânia Bonfim dos Santos \\ ORCID: https://orcid.org/0000-0002-8210-1774 \\ Universidade Federal do Pará, Brasil \\ E-mail: shirleyvania@yahoo.com.br \\ Mary Elizabeth de Santana \\ ORCID: https://orcid.org/0000-0002-3629-8932 \\ Universidade do Estado do Pará, Brasil \\ E-mail: betemary@terra.com.br
}

\begin{abstract}
Resumo
Objetivo: caracterizar as evidências científicas sobre as estratégias de promoção à saúde dos trabalhadores de enfermagem na pandemia da Covid-19. Metodologia: trata-se de estudo de revisão integrativa que tem por escopo caracterizar as evidências disponíveis na literatura sobre as estratégias de contenção do acometimento da saúde dos trabalhadores de Enfermagem durante a pandemia do Covid-19. Resultados: foram selecionados seis ( $n=6)$ estudos, dentre os quais cinco $(n=5)$ foram produzidos no Brasil e publicados em periódicos nacionais, apenas um artigo encontrado foi de origem internacional, quanto aos níveis de evidência, todos os artigos inclusos nesta revisão foram classificados com nível de evidência V, por possuírem a abordagem descritiva como método. Conclusão: os estudos incluídos nessa revisão vêm a caracterizar as dificuldades encontradas por enfermeiros no cenário da pandemia, bem como as estratégias adotas por organizações (a exemplo de sindicatos), das instituições hospitalares, ou até mesmo da sensibilidade de outros profissionais integrantes da equipe multiprofissional, para o cuidado físico e psicológico da equipe de enfermagem. Espera-se que os resultados deste estudo venham a contribuir com a reflexão, reprodução e com o estímulo destas estratégias e de novos estudos que abordem a cultura da saúde do trabalhador.
\end{abstract}

Palavras-chave: Infecções por coronavírus; COVID-19; Saúde do trabalhador; Enfermagem.

\begin{abstract}
Objective: to characterize the scientific evidence on the health promotion strategies of nursing workers during the Covid-19 pandemic. Methodology: this is an integrative review study that aims to characterize the evidence available in the literature on strategies to contain the health of nursing workers during the Covid-19 pandemic. Results: six $(n=6)$ studies were selected, among which five $(n=5)$ were produced in Brazil and published in national journals, only one article found was of international origin, regarding the levels of evidence, all articles included in this review, they were classified as evidence level V, as they have the descriptive approach as a method. Conclusion: the studies included in this review come to characterize the difficulties encountered by nurses in the pandemic scenario, as well as the strategies adopted by organizations (such as unions), hospital institutions, or even the sensitivity of other professionals in the multidisciplinary team, for the physical and psychological care of the nursing team. It is hoped that the results of this study will contribute to the reflection, reproduction and encouragement of these strategies and new studies that address the culture of worker's health.
\end{abstract}

Keywords: Coronavirus infections; COVID-19; Worker's health; Nursing.

\section{Resumen}

Objetivo: caracterizar la evidencia científica sobre las estrategias de promoción de la salud de los trabajadores de enfermería durante la pandemia de COVID-19. Metodología: se trata de un estudio de revisión integradora que tiene como objetivo caracterizar la evidencia disponible en la literatura sobre estrategias para contener la salud de los trabajadores de enfermería durante la pandemia Covid-19. Resultados: se seleccionaron seis $(n=6)$ estudios, de los cuales cinco $(n=5)$ fueron producidos en Brasil y publicados en revistas nacionales, solo un artículo encontrado fue de origen internacional, en cuanto a los niveles de evidencia, todos los artículos incluidos en este revisión, se clasificaron como nivel de evidencia V, ya que tienen el enfoque descriptivo como método. Conclusión: los estudios incluidos en esta revisión vienen a caracterizar las dificultades encontradas por las enfermeras en el escenario 
pandémico, así como las estrategias adoptadas por organizaciones (como sindicatos), instituciones hospitalarias, o incluso la sensibilidad de otros profesionales del equipo multidisciplinario. para el cuidado físico y psicológico del equipo de enfermería. Se espera que los resultados de este estudio contribuyan a la reflexión, reproducción y estímulo de estas estrategias y nuevos estudios que abordan la cultura de la salud del trabajador.

Palabras clave: Infecciones por coronavirus; COVID-19; Salud del trabajador; Enfermería.

\section{Introdução}

O enfrentamento da pandemia do novo coronavírus, fez com que pudéssemos voltar nossos olhos para a Política de Saúde dos Trabalhadores, em especial aos trabalhadores de saúde, os quais estiveram desde o início da pandemia mais suscetíveis a serem contaminados por causa da frequente exposição ao vírus e tendo uma escassez de recursos, materiais e equipamento de proteção individual (EPI) para suprir a grande demanda de pacientes infectados (Lima et al., 2020; Cabello \& Pérez, 2020). A legislação trabalhista brasileira, via Norma Regulamentadora de Segurança e Saúde no Trabalho em Serviços de Saúde (NR32), indica a obrigatoriedade do empregador fornecer ao trabalhador EPI em quantidade suficiente, descartáveis ou não, para o desenvolvimento seguro das tarefas de trabalho. Além do fornecimento, deve-se assegurar a capacitação de forma contínua e a garantia de proteção ao trabalhador, sempre que houver mudança das condições de exposição a agentes biológicos (Duarte et al, 2020).

Em se tratando de profissionais da saúde, no Brasil, os trabalhadores de enfermagem representam o maior contingente de trabalhadores da saúde, dentre os quais estão distribuídos em 565.458 enfermeiros, 1.320.239 técnicos de enfermagem e 419.959 auxiliares de enfermagem (Conselho Federal de Enfermagem, 2020). Pereira et al (2020) destacam por meio de revisão de literatura os desafios de enfermeiros no contexto hospitalar frente a pandemia da Covid-19, podendo-se dar destaque a altas cargas de trabalho, necessidade de se readequar as formas de atendimento, medidas de biossegurança, bem como no enfrentamento aos altos índices de óbito durante os plantões, gerando exaustão física e metal e frustação, o que ocasiona sentimento de impotência e insegurança profissional.

Até o final de julho, de 2020, 173.440 casos de síndrome gripal (SG) foram confirmados para a Covid-19 em profissionais da área da saúde no Brasil. As profissões com maior registro de casos foram os técnicos ou auxiliares de enfermagem (59.635), seguido dos enfermeiros (25.718), em relação aos casos graves da doença de Covid-19, que necessitaram de internação hospitalar, foram confirmados 697 casos (Conselho Federal de Enfermagem, 2020). Neste cenário, a classe dos profissionais de Enfermagem enfrenta importantes dilemas, principalmente no que diz respeito a condições de trabalho e materiais adequados para a proteção individual. Durante a pandemia, notou-se o aparecimento de lesões e erosões na pele dos trabalhadores, devido a manipulação incorreta das máscaras de proteção e o seu uso prolongado, e os elevados índices de infecção e mortalidade de profissionais de enfermagem pela Covid-19 (Miranda et al., 2020).

Com as evidências mundiais de impactos causados pela pandemia da Covid-19 em trabalhadores de enfermagem, constata-se a necessidade de investigação e ações referentes à saúde física e mental destes profissionais durante este período, tendo em vista que estes estão sendo submetidos a carga elevada de experiências e emoções negativas requerem cuidados e atenção, e mediante a isso, o estudo em evidência é essencial para somar na produção de mais informações que possam viabilizar medidas profiláticas a estes trabalhadores.

\section{Metodologia}

Trata-se de estudo de revisão integrativa que tem por escopo resumir as pesquisas já realizadas e estabelecer a partir de uma avaliação crítica com a meta de sintetizar e analisar os dados para desenvolver uma explicação mais ampla de um fenômeno específico, com a meta de síntese e análise dos achados nos estudos (Mendes; Silveira \& Galvão, 2008). 
Adotou-se as seis etapas para construção desta revisão: seleção da pergunta de pesquisa; definição dos critérios de inclusão e exclusão de estudos e seleção da amostra; estudos selecionados; considerando as características; análise crítica dos achados; interpretação dos resultados e reportar, de forma clara, a evidência identificada (Whittemore \& Knafl, 2005).

Neste estudo foi adotada a estratégia PICO, para mediar a construção da pergunta norteadora da revisão, os componentes da estratégia para a construção da pergunta são $\mathrm{P}$ (profissionais de enfermagem) I (estratégias de promoção à saúde) C (pandemia do Covid-19) O (saúde do trabalhador), dando origem a questão "Quais são as evidências disponíveis na literatura sobre as estratégias de promoção à saúde dos profissionais de Enfermagem na pandemia da Covid-19?"

\subsection{Critérios De Inclusão E Exclusão}

Serão incluídos os estudos publicados em inglês, espanhol e português, sobre as estratégias adotadas para a promoção da saúde do trabalhador durante a pandemia da Covid-19.

Serão excluídos os artigos que estiverem fora do período e da temática estabelecidos na busca e estudos de revisão integrativa, aqueles que forem oriundos de outros estudos secundários, bem como não serão incluídos neste estudo materiais de "literatura cinzenta".

\subsection{Busca De Estudos}

Nesta etapa foram estabelecidas as bases de dados a serem utilizadas na busca das evidencias científicas e os Descritores em saúde (DECS, em se tratando da busca de produções nacionais e MESH para a busca de artigos internacionais) para mediar a busca da literatura, para tanto definiu-se a utilização dos seguintes descritores e o operador boleando OR (MESH): "Infecções por Coronavírus"; "Betacoronavírus"; "Saúde do Trabalhador"; O período temporal de busca definido foi de artigos publicados no período de 2020 a 2021. As buscas ocorreram nas seguintes bases de dados que são Literatura LatinoAmericana e do Caribe em Ciências da Saúde (LILACS); Medical Literature Analysis and Retrieval Sisten On-line (MedLine)/ PubMed (via National Library of Medicine); SCielo e BDENF. As estratégias de busca nas bases de dados se deram segundo a combinação dos descritores e podem ser vistas no Quadro 1.

Quadro 1. Estratégia de Busca nas Bases de Dados. Belém, Brasil, 2021.

\begin{tabular}{|c|c|}
\hline MEDLINE/PUBMED & $\begin{array}{l}\text { (("COVID-19"[Mesh]) OR "Occupational Health"[Mesh] OR “Occupational Safety" OR } \\
\text { "Employee Health” AND "Nurses"[Mesh] OR "Registered Nurses" }\end{array}$ \\
\hline BDENF/LILACS & $\begin{array}{l}\text { (tw: ("Infecções por coronavírus" OR "Betacoronavírus" OR "Saúde do Trabalhador", } \\
\text { "Enfermagem" OR "Nursing" AND "Nurses" OR "Occupational Health" AND } \\
\text { "Occupational Safety" AND "COVID-19" OR "Salud Laboral" AND Salud de los } \\
\text { Trabajadores OR COVID-19 AND "coronavirus" AND "SARS-CoV-2" =(db: ("LILACS" } \\
\text { OR "BDENF"). }\end{array}$ \\
\hline SCIELO & $\begin{array}{l}\text { "Infecções por coronavírus" OR "Betacoronavírus" OR "Saúde do Trabalhador", } \\
\text { "Enfermagem" AND "Saúde dos Trabalhadores" AND "Atenção à Saúde do Trabalhador" } \\
\text { AND "Enfermeiros", "Infecção pelo SARS-CoV-2" AND "COVID-19" }\end{array}$ \\
\hline CINAHL & $\begin{array}{l}\text { (("Nurses,Nurse, Nursing"[Mesh]) AND "Covid-19" AND "Occupational Health"[Mesh] } \\
\text { OR "Cross Infecction Prevent and Control" OR "Occupational Safety" OR "Employee } \\
\text { Health" AND "Nurses"[Mesh] OR "Registered Nurses" }\end{array}$ \\
\hline
\end{tabular}

Fonte: Autores. 
Após a coleta de dados os dados foram organizados e filtrados em um software online (Rayyan) que auxilia na filtração dos dados coletados.

\subsection{Análise Dos Dados}

Mediante a seleção e filtragem dos artigos encontrados na busca realizada na etapa anterior do estudo, os dados do processo são apresentados por intermédio de fluxograma explicativo (Hopia et al., 2016; Galvão, 2015), segundo as recomendações do protocolo PRISMA, o qual pode ser visualizado na Figura 1. A análise realizada em sete níveis: Nível I (Estudos de revisão sistemática ou metanálise de ensaios clínicos randomizados controlados); Nível II (Ensaio clínico randomizado controlado bem delineado); Nível III (Estudos de ensaios clínicos bem delineados sem randomização); Nível IV (Estudos de corte e de caso-controle bem delineados (não experimentais); Nível V (Estudos de revisão sistemática de estudos descritivos e qualitativos); Nível VI (Evidências derivadas de um único estudo descritivo ou qualitativo); Nível VII (Evidências oriundas de opinião de autoridades ou comitês) (Melnyk, 2010).

Figura 1. Fluxograma dos Cruzamentos e Resultados de Busca, segundo as recomendações do protolo PRISMA. Belém, Brasil, 2021.

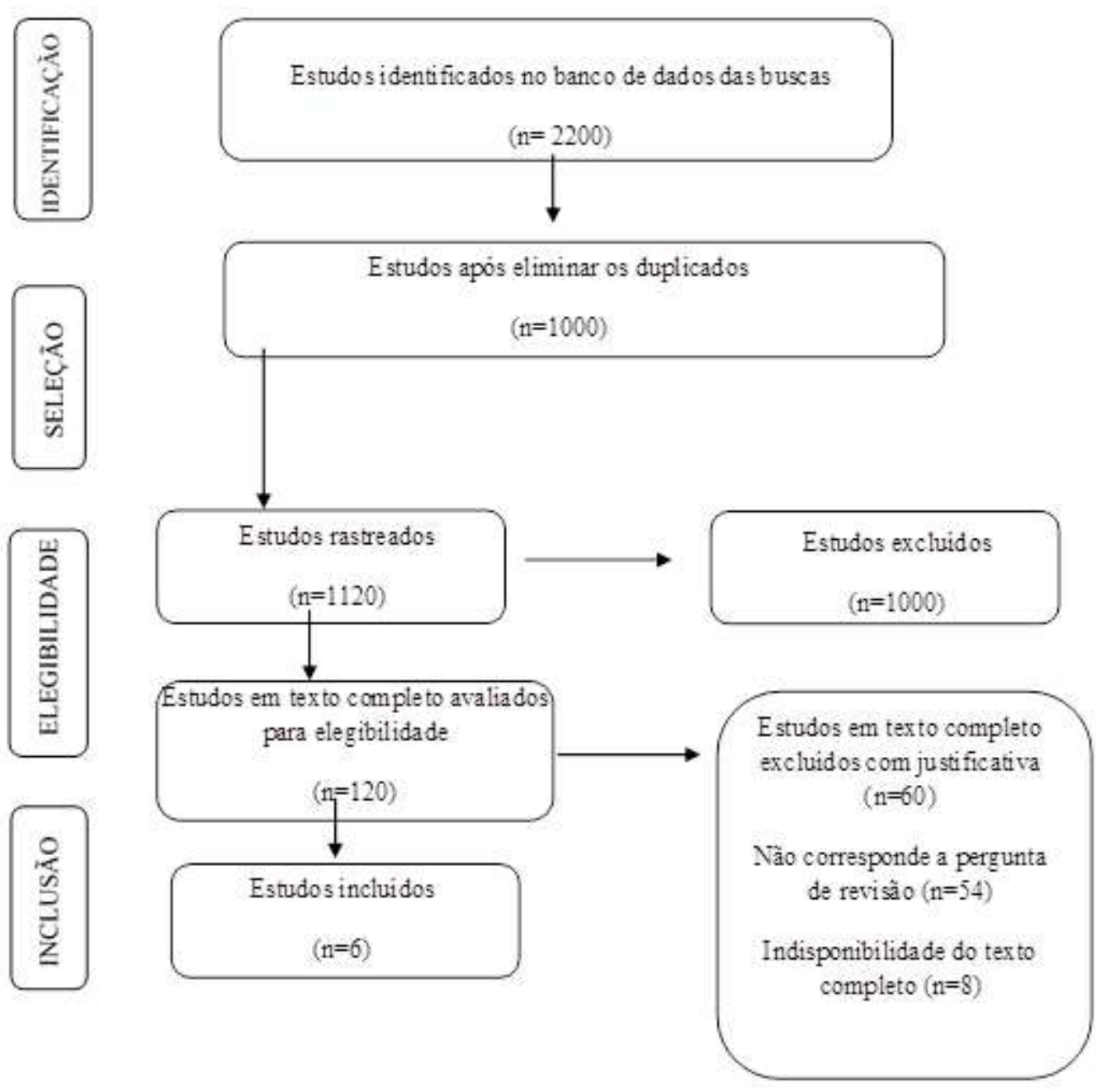

Fonte: Autores. 


\section{Resultados e Discussão}

Foram incluídos seis $(\mathrm{n}=8)$ estudos nesta revisão, estes foram compreendidos no período de 2020 a 2021 tendo sua maioria no ano de 2020 (por conta da atualidade do tema em estudo), quanto aos níveis de evidência verificou-se a predominância de estudos descritivos (nível de evidência VI), o que aponta para uma maior necessidade de produção científica diante da temática com maiores níveis de evidência, para que se conheçam os resultados de experiências exitosas na segurança laboral de profissionais da enfermagem na pandemia da Covid-19.

No Quadro 2 estão apresentados os dados dos estudos analisados segundo as variáveis: autores, ano de publicação, nível de evidência. Em seguida no Quadro 3 podem ser encontradas as principais estratégias encontradas na literatura a respeito da segurança do trabalhador de Enfermagem na pandemia do Covid-19.

Quadro 2. Estudos encontrados conforme autoria, ano de publicação, nível de evidência.

\begin{tabular}{|c|c|c|c|c|}
\hline AUTORIA & $\begin{array}{l}\text { PAÍS DE ORIGEM E } \\
\text { ANO DE } \\
\text { PUBLICAÇÃO }\end{array}$ & OBJETIVOS & $\begin{array}{l}\text { NÍVEL DE } \\
\text { EVIDÊNCIA }\end{array}$ & $\begin{array}{l}\text { BASE DE } \\
\text { DADOS }\end{array}$ \\
\hline $\begin{array}{l}\text { TRIGUEIRO } \\
\text { et al. }\end{array}$ & $\begin{array}{c}\text { Brasil } \\
2020\end{array}$ & $\begin{array}{l}\text { Relatar o uso de auriculoterapia na otimização da } \\
\text { saúde de trabalhadores de urgência na pandemia da } \\
\text { Covid-19 }\end{array}$ & Nível VI & SCIELO \\
\hline $\begin{array}{l}\text { ARAÚJO- } \\
\text { DOS-SANTOS } \\
\text { et al. }\end{array}$ & $\begin{array}{c}\text { Brasil } \\
2020\end{array}$ & $\begin{array}{l}\text { Relatar a experiência do Comitê de Enfermagem } \\
\text { para Enfrentamento da Covid-19 na Bahia }\end{array}$ & Nível VI & SCIELO \\
\hline $\begin{array}{l}\text { GALLASCH } \\
\text { et al. }\end{array}$ & $\begin{array}{c}\text { Brasil } \\
2020\end{array}$ & $\begin{array}{l}\text { Descrever as principais recomendações sobre ações } \\
\text { de prevenção de contágio relacionadas à exposição } \\
\text { ocupacional dos profissionais de saúde atuantes } \\
\text { frente à Covid-19 }\end{array}$ & Nível VI & SCIELO \\
\hline TOBASE et al. & $\begin{array}{l}\text { Brasil } \\
2021\end{array}$ & $\begin{array}{l}\text { Refletir sobre a utilização da escuta empática como } \\
\text { estratégia de acolhimento aos profissionais de } \\
\text { enfermagem no enfrentamento dos desafios durante } \\
\text { a pandemia pelo novo coronavírus }\end{array}$ & Nível VI & SCIELO \\
\hline BRAGA et al. & $\begin{array}{l}\text { Brasil } \\
2020\end{array}$ & $\begin{array}{l}\text { Descrever a construção e validação de um checklist, } \\
\text { para paramentação e desparamentação de EPIs }\end{array}$ & Nível VI & $\begin{array}{l}\text { LILACS/BDE } \\
\text { NF }\end{array}$ \\
\hline NEWBY et al. & $\begin{array}{l}\text { EUA } \\
2020\end{array}$ & $\begin{array}{l}\text { Compartilhar informações úteis para a proteção de } \\
\text { trabalhadores de enfermagem durante a pandemia de } \\
\text { Covid-19. }\end{array}$ & Nível VI & $\begin{array}{l}\text { PUBMED/ME } \\
\text { DILNE }\end{array}$ \\
\hline $\begin{array}{l}\text { DINCERA \& } \\
\text { INANGIL }\end{array}$ & $\begin{array}{c}\text { Turquia } \\
2020\end{array}$ & $\begin{array}{l}\text { Investigar a eficácia a aplicação online de Técnicas } \\
\text { de Liberdade Emocional (TLE) para a prevenção do } \\
\text { estresse, ansiedade e burnout em enfermeiras } \\
\text { envolvidas no cuidado de pacientes com COVID. }\end{array}$ & Nível II & CINAHL \\
\hline
\end{tabular}

Fonte: Autores. 
Quadro 3. Resultados dos estudos selecionados.

\begin{tabular}{|c|c|}
\hline AUTORIA & $\begin{array}{l}\text { PRINCIPAIS AÇÕES DE CONTENÇÃO DO ACOMENTIMENTO À SAÚDE DE } \\
\text { TRABALHADORES DE ENFERMAGEM DURANTE A PANDEMIA DO COVID-19 }\end{array}$ \\
\hline TRIGUEIRO et al. & $\begin{array}{l}\text { A auriculoterpia tem potencial para contribuir no enfrentamento de situações físicas e psicoemocionais } \\
\text { dos trabalhadores atuantes no combate à pandemia Covid-19. Os participantes requisitaram a manutenção } \\
\text { das sessões de auriculoterapia no contexto atual e no pós-pandemia. }\end{array}$ \\
\hline $\begin{array}{l}\text { ARAÚJO-DOS-SANTOS } \\
\quad \text { et al. }\end{array}$ & $\begin{array}{l}\text { A identificação das demandas, dúvidas e casos de contaminação dessas/es trabalhadoras/es subsidiam } \\
\text { ações para a assistência segura. Além disso, é fortalecida a capacidade de atuação política e técnica das } \\
\text { entidades e órgãos envolvidos, o que é útil para as lutas das/os trabalhadoras/es após a pandemia }\end{array}$ \\
\hline GALLASCH et al. & $\begin{array}{l}\text { A garantia de acesso a equipamentos de proteção individual em número suficiente e com eficácia } \\
\text { reconhecida são elementos essenciais destacados no estudo. }\end{array}$ \\
\hline TOBASE et al. & $\begin{array}{l}\text { Como estratégia de acolhimento para o fortalecimento dos profissionais de enfermagem, a escuta } \\
\text { empática é recurso potente, especialmente durante o enfrentamento da Covid-19. }\end{array}$ \\
\hline BRAGA et al. & $\begin{array}{l}\text { A utilização do checklist de EPI validado na prática clínica poderá auxiliar na realização da paramentação } \\
\text { e desparamentação dos EPI, com maior segurança, contribuindo, assim, para a prevenção de } \\
\text { autocontaminação pelo SARS-CoV-2, pelos profissionais de enfermagem que prestam assistência a } \\
\text { pacientes com suspeita ou confirmação de Covid-19, bem como em outros contextos na assistência a } \\
\text { pessoas com doenças infectocontagiosas. }\end{array}$ \\
\hline NEWBY et al. & $\begin{array}{l}\text { Foram utilizadas estratégias de redução de tráfego de profissionais de enfermagem nas unidades de } \\
\text { terapia intensiva (estipulou-se um limite de abertura da porta, uma área de pausa para a higienização, um } \\
\text { fluxo exclusivo para transporte de pacientes com Covid etc.) }\end{array}$ \\
\hline DINCERA \& INANGIL & $\begin{array}{l}\text { A intervenção online, de sessão única e utilizando a TLE foi eficaz na redução significativa do estresse, } \\
\text { ansiedade e esgotamento. Embora várias perguntas permaneçam sem resposta em relação a esta técnica, } \\
\text { como por exemplo, a durabilidade desses benefícios. Esta intervenção é rápida, fácil de fornecer e pode } \\
\text { ser aplicada a enfermeiras que cuidam de pessoas com COVID-19 em todo o mundo. }\end{array}$ \\
\hline
\end{tabular}

Fonte: Autores.

Os principais resultados dos estudos selecionados nesta revisão foram organizados segundo similaridade semântica e derem origem a duas categorias temáticas de discussão, apresentadas a seguir:

\section{Estratégias Utilizadas para Garantir a Seguridade Física de Trabalhadores de Enfermagem na Pandemia da Covid-19}

A efetividade das políticas públicas para o enfrentamento de uma pandemia é essencial, não apenas para a população geral ou o grupo de risco, pois deve englobar os profissionais de saúde e as instituições hospitalares (Oliveira, 2020). No que diz respeito as medidas de contenção de riscos de contaminação de profissionais da saúde pelo novo coronavírus os estudos de Araújo-Dos-Santos (2020), Gallasch (2020), Braga (2020) e Newby (2020) descrevem as experiências que tiveram no contexto da assistência durante a pandemia, e principalmente quais estratégias adotaram para a proteção dos trabalhadores de enfermagem.

Araújo-Dos-Santos (2020) relatou a experiencia sobre a criação e o trabalho desenvolvido por um comitê de Enfermagem para Enfrentamento da Covid-19 na Bahia. Este comitê foi criado em virtude do medo vivenciado por trabalhadoras/ es em Enfermagem, que se sentiam pouco apoiadas/os e com diversas dúvidas sobre a pandemia e de como enfrentá-la. Para tanto, foram criados 5 grupos de trabalho: a responsabilidade destes grupos era realizar articulações com Secretarias de Saúde, Ministério Público, Ministério Público do Trabalho, Vigilância de Saúde do Trabalhador, dentre outros órgãos; monitorar o canal virtual criado para receber e acolher demandas, dúvidas e denúncias de trabalhadoras/es em enfermagem, bem como pela produção de materiais educativos para os trabalhadores de enfermagem; elaboração dos planos de contingência e revisa os fluxos e protocolos criados pela Secretaria Municipal de Saúde; orientação específica das trabalhadoras em ILP, pois essas estão em contato direto com um/ dos grupos vulneráveis ao Coronavírus; realizar visitas a serviços de saúde para a fiscalização das condições de saúde e de segurança das/os trabalhadoras/es; rastrear e acompanhar os casos de contaminação por coronavírus entre trabalhadoras/es em enfermagem. 
Em relação a contenção de contaminações relacionadas a exposição ocupacional dos profissionais de enfermagem Gallasch (2020) e Braga (2020) apresentam similaridade em relação a esta temática, uma vez que Gallasch (2020) vem a apontar e caracterizar as necessidades de proteção dos profissionais de Enfermagem e Braga (2020) vem a sugerir um checklist de paramentação e desparamentação a ser utilizado pela equipe de enfermagem durante o cuidado de pessoas com Covid.

No estudo de Gallasch (2020) apontou-se que para reduzir a transmissão do patógeno nos serviços de saúde, devem ser previstas práticas de prevenção mesmo antes da chegada do paciente na unidade, como reagendando dos atendimentos eletivos ou realizando entrevista e orientações prévias sobre os seus sintomas. Caso não seja possível um contato anterior, deve-se prezar por ações pré-triagem. Outra medida protetiva adota está relacionada a restrição de portas de entrada, limitando o tipo de atendimento na unidade, o fornecimento de máscara com rápido isolamento de casos suspeitos, reduzindo o contato com os demais pacientes e profissionais, ou seja, é necessário garantir a correta triagem dos casos da Covid-19 para reconhecimento precoce e controle da fonte, de forma a isolar os pacientes sob suspeita de infecção e aplicar precauções recomendadas. Desse modo, vindo a contribuir com a redução da transmissão comunitária, podendo levar a uma redução de contaminados buscando pelo serviço de saúde, consequentemente diminuindo a probabilidade de exposição dos trabalhadores alocados nestas unidades.

Além destas práticas Gallash (2020) cita o automonitoramento como medida preventiva, uma vez que por meio da regulação dos expostos ao risco, por meio da identificação da ocorrência de febre ou sintomas respiratórios (tosse seca, dor de garganta e falta de ar) que podem ser um sinal de alerta quanto à Covid-19, deve-se alertar aos gestores dos serviços de saúde sobre a necessidade de desenvolvimento de um plano de ação estabelecendo fluxo de condutas, para a proteção e cuidado de seus servidores.

Em se tratando de medidas protetivas para a biossegurança, a ANVISA é o órgão responsável pela regulamentação destas medidas e na pandemia da covid-19 recomendou a adoção de higiene das mãos com água e sabonete líquido ou preparação alcoólica a 70\%, utilização de proteção ocular e/ou facial, máscara cirúrgica, avental impermeável e luvas de procedimento; para uma rotina onde há a geração de aerossóis ( a exemplo de intubação ou aspiração traqueal, bem como em situações de ressuscitação cardiopulmonar, ventilação manual, e cuidado de pacientes críticos, deve-se utilizar além destas medidas protetivas citadas, gorro e máscara N95 ou FFP2. Quanto às equipes de apoio hospitalar (aqueles profissionais os quais não estão diretamente atuando na assistência), recomenda-se a higienização das mãos, óculos de proteção ou protetor facial, a utilização de máscara cirúrgica, avental impermeável e luvas de procedimento (Brasil,2020).

Com a crescente incidência de casos de covid no país, o acesso aos EPI para profissionais de saúde tem se tornado um grande receio, em virtude da possibilidade de escassez destes produtos no mercado e nas instituições hospitalares com alta demanda de atendimento e com a crise sociopolítica e econômica causada pelos impactos da pandemia. em um cenário como este, o fornecimento para as equipes de saúde deve ser priorizado e impõe o uso racional dos insumos para evitar a impossibilidade técnica de prestar cuidados aos pacientes com Covid-19, pelo risco iminente de exposição ao vírus.

A legislação trabalhista brasileira, via norma regulamentadora de segurança e saúde no trabalho em serviços de saúde (nr32), indica a obrigatoriedade de o empregador prover ao trabalhador epi em quantidade suficiente, descartáveis ou não, que sejam necessários para o desenvolvimento seguro das tarefas de trabalho. além do fornecimento, deve-se assegurar a capacitação de forma contínua e a garantia de proteção ao trabalhador sempre que houver mudança das condições de exposição a agentes biológicos (Who,2020).

No que diz respeito ao desenvolvimento seguro das tarefas de trabalho Braga (2020) traz uma tecnologia, em formato de checklist, como promotora da segurança dos profissionais de enfermagem durante o cuidado de pessoas com Covid-19. Para tanto, utilizou de um percurso metodológico divido sob as seguintes fases: revisão narrativa da literatura sobre as técnicas de paramentação e desparamentação dos EPI por profissionais de saúde; Elaboração de um roteiro e gravação de um vídeo sobre 
as técnicas de paramentação e desparamentação dos EPI; Treinamento em serviço sobre as técnicas de paramentação e desparamentação dos EPI com, aproximadamente, 500 profissionais de saúde de quatro instituições (atenção primária, secundária e terciária) de um município de Minas Gerais, no período de março a maio/2020 e implementação das referidas técnicas nos serviços pelos autores do presente estudo.

$\mathrm{O}$ checklist permite que a conferência dos passos a serem realizados em um determinado procedimento possam auxiliar na execução integral da prática, independentemente da falibilidade da memória da equipe, reforçando a verificação constante e incentivando a disciplina com alto desempenho (Tang, 2014).

O conteúdo do checklist proposto por Braga (2020) passou por processo de validação, juntamente a 20 profissionais especialistas na temática, do checklist dos EPI obteve concordância superior a 80\% (concordo e concordo totalmente) em todos os itens avaliados pelo comitê de especialistas. Apesar das sugestões não serem uniformes e a maioria descrita por um único especialista, elas foram analisadas no grupo focal on-line no formato síncrono, tendo em vista a pertinência. Todos os participantes do grupo focal $(n=6 ; 100 \%)$ concordaram com as sugestões dos especialistas, as quais foram incluídas na versão final da tecnologia.

O uso do checklist apresentado poderá contribuir com a redução de custos organizacionais por favorecer o uso correto dos EPI e minimizar o risco de contaminação entre profissionais, o que resulta em uma equipe disponível para a assistência e menores índices de afastamentos do trabalho. Além disso, o instrumento validado poderá auxiliar os profissionais na identificação de fragilidades em cada uma das etapas, no processo de paramentação e desparamentação dos EPI nos contextos da atenção primária, secundária e terciária à saúde e fornecer indicadores de avaliação, apontando a necessidade de revisão, adequação ou capacitação para melhorar a segurança (Braga, 2020).

Newby (2020), sugere como medidas de prevenção do risco de adoecimento ocupacional durante a pandemia: O uso de EPIs segundo as recomendações da OMS de paramentação e desparamentação, por meio do uso criterioso dos recursos pode-se estender a janela de proteção para os profissionais da linha de frente. Outro fator citado pelos autores diz respeito a sistematização da assistência prestada pela equipe, de modo a agrupar atividades e reorganizar o espaço para o monitoramento dos pacientes fora da área de isolamento, por meio da externalização dos monitores de parâmetros ventilatórios e de sinais vitais para fora das áreas de isolamento, e do controle do fluxo de pacientes infectados ou sintomáticos respiratórios nas áreas do hospital, além disso adotou-se como estratégia um limite de abertura das portas para que fossem reduzidas as chances de saída do "ar contaminado" presentes nas salas de isolamento. A videoconferência e os estetoscópios digitais foram tecnologias adotadas pelos enfermeiros como forma de controle infecções ocupacionais, por permitir que a equipe avalie o paciente sem estar na sala. Em alguns casos, utilizava-se a videochamada para compartilhar notícias com a família para reduzir ainda mais as chances de transmissão comunitária do vírus.

As estratégias de cuidados com a equipe de enfermagem expostas em Newby (2020) ao serem comparados com as estratégias adotadas nos estudos de Araújo-Dos-Santos (2020), Gallasch (2020) e Braga (2020), refletem a realidade da saúde dos trabalhadores em diferentes contextos econômicos, sociais e políticos entre as possibilidades para os enfermeiros de um país da América do Norte e aqueles que atuaram durante a pandemia em um país da América Latina. Apesar de ser uma realidade mundial, a escassez de EPI's não se apresenta como um fator problemático na pesquisa de Newby (2020), o que pode ser reflexo da detenção dos meios de produção e de retenção de capital dos Estados Unidos, outro fator a ser considerado é de que as estratégias voltadas aos profissionais de enfermagem em Newby (2020) foram oriundas de processos da instituição de saúde onde os pesquisadores atuavam, quando comparadas as estratégias adotadas no Brasil segundo as informações presentes nos estudos de Araújo-Dos-Santos (2020), Gallasch (2020) e Braga (2020) notou-se que a falta de recursos de proteção individual e de promoção a saúde do trabalhador eram realidades encontradas no país, o que por conseguinte, levou instituições como conselhos, sindicatos, ONG's e até mesmo iniciativas entre os próprios profissionais de enfermagem e da 
equipe multiprofissional a adotarem estratégias para arrecadação de EPI's e adoção de outras medidas as quais viessem a promover a saúde destes profissionais na pandemia.

Com relação às respostas dadas via políticas sociais no cenário mundial, a pandemia tem tido um melhor encaminhamento nos países que preservaram os serviços públicos de caráter universal. Ou seja, a presença do Estado nas respostas às demandas da população determina o grau de eficiência observado no combate ao novo coronavírus, na maioria das situações. Neste momento de agudização da crise, mais do que nunca, torna-se explícito a necessidade de garantia da seguridade social, sobretudo a saúde e a assistência social - representados enquanto políticas sociais no Sistema Único de Saúde (SUS) e no SUAS - são (ou deveriam ser) consideradas as linhas de frente de enfrentamento à esta crise, seja pela oferta de cuidados em saúde, seja pela oferta de atendimentos socioassistenciais ou pela transferência de recursos financeiros que garantam a subsistência de sujeitos que têm as suas condições socioeconômicas agravadas por esta situação (Bardi et al., 2020).

De acordo com o Observatório de Política Fiscal, ligado ao Instituto Brasileiro de Economia (Ibre), da Fundação Getúlio Vargas, o Brasil tem ficado atrás de outros países no que se refere às medidas de combate à expansão do novo coronavírus, investindo apenas pouco mais de $2 \%$ do Produto Interno Bruto (PIB), ante 17\% em outros países, o que representa cerca de um décimo da média dos países no combate à propagação da Covid-19. A precariedade e a insuficiência das respostas via políticas sociais têm desencadeado ações de auto-organização da classe trabalhadora que luta pela própria sobrevivência. Observa-se neste período, o engajamento de ONGs e das associações de bairros em iniciativas de distribuição de alimentos, produtos de limpeza e máscaras, ações que a longo prazo não resolvem o problema, mas que, neste momento, minimizam os efeitos deletérios dessa crise (Antunes, 2020; Pires, 2020).

\section{Estratégias Utilizadas para Garantir a Seguridade Psicológica de Trabalhadores de Enfermagem durante a Pandemia do Covid-19.}

Na pandemia, a necessidade de maiores demandas de cuidado do profissional no atendimento integral em numerosos casos de urgência e gravidade, pessoas das mais diferentes faixas etárias com prognósticos desfavoráveis, gerou angústia, frustração e exaustão emocional na luta pela vida. Ademais, ressalta-se que as longas horas de trabalho provocam estresse ocupacional (cansaço físico e psicológico), redução do desempenho das atividades de cuidado, riscos de transtornos depressivos e ansiosos, e aumento do absenteísmo (Bordignon, 2016).

Neste contexto, Tobase (2021) e Trigueiro (2020) abordaram estratégias que visassem garantir a seguridade psicológica dos trabalhadores de Enfermagem face ao estresse ocupacional causado diante da sobrecarga de trabalho e emocional desta classe profissional no contexto da assistência de pacientes com Covid-19, para tanto foram utilizadas como ferramentas de promoção de saúde psicoemocional a auriculoterapia e escuta empática.

A estratégia citada por Tobase (2021) para o acolhimento dos profissionais durante a pandemia foi a escuta empática, cuja busca trazer a consciência, com afeto, respeito, empatia e generosidade, para a transformação dessas relações, uma vez que os aspectos estruturais, inadequação das condições de trabalho, escassez de recursos, significativo aumento de óbitos durante os plantões, e fora deles, no contexto familiar, social e profissional, abalaram profundamente os profissionais da saúde, em especial os enfermeiros.

A escuta empática tem sido muito utilizada diante da atual pandemia como estratégia para o fortalecimento emocional. Frente à necessidade de acolhimento e suporte no enfrentamento de violências vivenciadas, o indivíduo se sente considerado, compreendido em suas vulnerabilidades e fortalecido para identificar suas potencialidades (Tobase, 2021).

A escuta empática também é valiosa para identificar pessoas vulneráveis do ponto vista psicossocial. Ofertar acolhimento e empatia fortalece o profissional, mesmo em demandas indiretamente relacionadas ao trabalho. Grupos de 
voluntários qualificados se solidarizam com os desafios da categoria, promovendo espaços online gratuitos de escuta empática para acolhimento da enfermagem, o que contribui, positivamente, ao ressignificar suas demandas, percebendo-se mais fortalecido e valorizado como pessoa e profissional (Tobase, 2021).

No cotidiano, o apoio entre os pares é essencial e diante da identificação do estresse ocupacional. Promover acolhimento e escuta é imprescindível para que os integrantes da equipe se sintam encorajados a identificar seus sentimentos e necessidades, por meio da expressão de suas demandas (Bordignon, 2016).

No que diz respeito ao apoio entre pares, o estudo de Trigueiro (2020) descreveu uma ação de suporte aos profissionais da enfermagem, promovidos por fisioterapeutas por meio da prática da auriculoterapia. A utilização de Práticas Integrativas e Complementares em Saúde (PICS) como terapêutica potencializadora do cuidado tem sido valorizada e implementada no Sistema Único de Saúde (SUS) brasileiro (Sousa, 2017).

Dentre as PICS, a auriculoterapia (ramo da Acupuntura) destaca-se por realizar a estimulação mecânica de zonas específicas do pavilhão auricular, cujos possuem pontos reflexos correspondentes a órgãos e funções corpóreas. Quando esses pontos são estimulados, é desencadeada uma série de fenômenos no cérebro que auxiliam no processo de cura. Promovendo assim, a analgesia e tratamento de diferentes afecções físicas e psicológicas (Souza, 2017).

Para tanto, foi realizada busca ativa dos trabalhadores no repouso desses profissionais e no pátio da base do serviço. Alguns buscaram espontaneamente as sessões de auriculoterapia por indicação de outros trabalhadores ou por convite feito anteriormente pela pesquisadora. Para essas sessões, as pessoas se dirigiam à biblioteca do serviço e lá eram convidadas individualmente a sentarem-se em cadeiras a uma distância de aproximadamente 1,50 metro entre eles. Eles também eram solicitados a aguardar sua vez para o atendimento, usavam máscara cirúrgica, e enquanto aguardavam, participavam de um momento inicial de meditação guiada pela psicóloga. Na sala, havia um refletor multicolorido e um difusor contendo óleo essencial de lavanda, enquanto tocava música, para a promoção do relaxamento. Todo o processo foi explicado passo a passo aos participantes para sua maior segurança e entendimento sobre a terapia. Após a terapêutica ter sido realizada em todos, foi feita breve explicação sobre a necessidade de manter as sementes postas na orelha no decorrer da semana. Todos foram convidados para dar continuidade à terapia nos dias de seus plantões, caso o desejassem (Trigueiro, 2020).

Tal experiência trouxe como pontos positivos: o fortalecimento de vínculos; melhoria da ambiência; melhoria do serviço prestado pelos profissionais, pois quem trabalha sem dor e tem uma boa qualidade do sono, torna-se mais produtivo; contribuição com a gestão, por fazer o colaborador se sentir cuidado e acolhido pelo serviço; sensação de empoderar e ajudar os colegas de trabalho em suas afecções físicas e emocionais. Os pontos negativos observados foram: impossibilidade de acolhimento de todos os profissionais do serviço, dada a carga horária de trabalho da especialista; ausência de local específico para realizar a terapia; falta de divulgação do serviço; ausência de um serviço institucionalizado para cuidar dos profissionais (Trigueiro, 2020).

A nível internacional, Dincera e Inangil (2020), utilizaram a técnica de liberdade emocional (TLE), cujo princípio básico consiste no envio sinais de ativação e desativação para o cérebro por meio de estímulos de pontos presentes na pele que têm propriedades elétricas. Esses pontos correspondem aos pontos de acupressão que, segundo a Medicina Tradicional Chinesa, regulam o fluxo das energias do corpo, acredita-se que equilibrar e harmonizar as energias do cliente relaxa e otimiza o corpo, a mente e as emoções (Moran, 2017).

Partindo deste princípio as pesquisadoras desenvolveram um ensaio clínico randomizado para avaliar a efetividade da técnica como uma estratégia de seguridade emocional e psicológica para trabalhadores de enfermagem que cuidam de pessoas com Covid-19. Antes de participar do estudo, os enfermeiros participantes do estudo foram randomizados para o grupo de intervenção ou controle. 
Após a conclusão do estudo, o TLE foi oferecido aos participantes do grupo de controle. A coleta de dados foi realizada online com o Survey Monkey e as entrevistas foram realizadas com o Zoom (Dincera \& Inangil,2020).

Foram incluídos 35 enfermeiros para o grupo de intervenção, os quais foram divididos em 7 subgrupos de 5 participantes. Para a intervenção, foi determinado um horário para a reunião em colaboração com os participantes de cada subgrupo, solicitou-se que durante a reunião os participantes permanecessem confortáveis em um ambiente o mais calmo e tranquilo possível durante a sessão, o tratamento foi mediado por um dos autores, que foi certificado para realizar a terapia, cada sessão durou em média 20 minutos. Para a avaliação da efetividade da terapia, os participantes eram submetidos a questionários de avaliação de burnout, depressão e ansiedade, antes e após cada sessão de TLE (Dincera \& Inangil,2020).

Como resultados da pesquisa de Dincera e Inangil (2020), observou-se que é desafio oferecer suporte para enfermeiros e outros profissionais de saúde impactados pela crise do COVID-19 e que a demanda de tempo para realizá-las já é uma fonte de estresse, por conta da demanda de cuidados intensivos de pessoas com Covid-19, portanto, encontrar tempo para a intervenção pode, por si só, contribuir para mais sofrimento. Além disso, as autoras descobriram que a FEL pode se apresentar como um tratamento eficaz e rápido para a redução de estresse, ansiedade e burnout.

Diferentemente do que diz respeito ao comparativo entre a disparidade da disponibilização de estratégias e materiais para a segurança física dos profissionais de enfermagem entre os países estrangeiros quando comparados a realidade brasileira, o cuidado com a saúde mental dos trabalhadores não foi tão diferente entre as realidades, uma vez que o suporte deficiente em relação a saúde mental de trabalhadores de enfermagem pode ser identificada nos estudos acima citados foi comum a ambas realidades, uma vez que todas as estratégias voltadas para a saúde destes trabalhadores teve sua origem na solidariedade entre os colegas de trabalho que compartilhavam das mesmas aflições, bem como da inquietação de pesquisadores os quais buscaram adaptar técnicas de medicina tradicional para a redução do estresse, burnout e depressão dos profissionais de enfermagem diante das cargas emocionais durante o cuidado de pessoas com Covid-19.

\section{Considerações Finais}

Desde o início da pandemia, os efeitos socioculturais, demográficos e econômicos têm sido relevantes para a incidência de mudanças de hábitos, comportamentos e do adoecimento psicológico da população em geral. Apesar disso, a população em geral pôde estar desenvolvendo suas atividades laborais no conforto do lar, o que por ventura, não foi a realidade dos profissionais de saúde, que incansavelmente compõe até o presente momento, a linha de frente no combate ao novo coronavírus, em se tratando dos profissionais de saúde, a enfermagem constitui o maior contingente da força de trabalho em saúde, e infelizmente houve significantes perdas e afastamento de profissionais desta classe, em virtude da pandemia, no entanto, apesar dessas adversidades, a classe vem demonstrando força para enfrentar e conter as consequências da Covid-19.

Nesse sentido, os estudos incluídos nessa revisão vêm a identificar algumas das dificuldades encontradas por enfermeiros no cenário da pandemia, e principalmente, quais foram as estratégias adotas, observou-se que grande parte dessas estratégias foram advindas de organizações (a exemplo de sindicatos, conselhos de classe e de grupos de pesquisa) ou até mesmo da sensibilidade de outros profissionais integrantes da equipe multiprofissional, para o cuidado físico e psicológico da equipe de enfermagem, em contrapartida a ações institucionalizadas pelos serviços de saúde ou por políticas públicas as quais visassem assistir estes profissionais em um cenário tão devastador quanto o da pandemia da Covid-19.

Quanto as limitações do estudo, pode-se justificar a inclusão de apenas sete estudos nesta revisão em virtude da atualidade da temática, assim como do pouco tempo que estes enfermeiros têm para produzir conteúdo científico em virtude das demandas de assistência, bem como e principalmente da deficiência da implementação de medidas de promoção e de prevenção da infecção por coronavírus pelos governantes e pelas instituições de saúde. Espera-se que os resultados deste 
estudo venham a contribuir com a reflexão, reprodução e com o estímulo destas estratégias e de novos estudos que abordem a cultura da saúde do trabalhador.

\section{Referências}

Antunes, R. (2020). O vilipêndio do coronavírus e o imperativo de reinventar o mundo. Tostes A; Filho HM. Quarentena: reflexões sobre a pandemia e depois, $1,181-88$.

Araújo-dos-Santos, T., Santos, H. S., Moraes, M. D. A., \& Mussi, F. C. (2020). Nursing Comiittee to Coping with COVID-19 in Bahia. Revista Brasileira de Enfermagem, 73.(2). https://doi.org/10.1590/0034-7167-2020-0469

Bordignon, M., \& Monteiro, M. I. (2016). Violence in the workplace in Nursing: consequences overview. Revista Brasileira de Enfermagem, 69, 996-999. https://doi.org/10.1590/0034-7167-2015-0133

Braga, L. M., Siman, A. G., Souza, C. C., Dutra, H. S., Gomes, A. P., \& Siqueira-Batista, R. (2020). Construção e validação do checklist para paramentação e desparamentação dos equipamentos de proteção individual. Revista de Enfermagem do Centro-Oeste Mineiro, 10. https://doi.org/10.19175/recom.v10i0.4079

Cabello, I. R., \& Pérez, I. R. (2020). El impacto de la pandemia por COVID-19 sobre la salud mental de los profesionales sanitarios. Coronavirus y Salud Publica. Escuela Andaluza de Salud Pública. https://doi.org/10.1101/2020.04.02.20048892

Conselho Nacional de Saúde. (2005). Princípios e diretrizes para a gestão do trabalho no SUS (NOB/RH-SUS). https://livroaberto.ibict.br/handle/1/891

Conselho Federal de Enfermagem.(2020). Observatório da Enfermagem [Internet]. http://observatoriodaenfermagem.cofen.gov.br/

Dincer, B., \& Inangil, D. (2021). The effect of Emotional Freedom Techniques on nurses' stress, anxiety, and burnout levels during the COVID-19 pandemic: A randomized controlled trial. Explore, 17(2), 109-114. https://doi.org/10.1016/j.explore.2020.11.012

Duarte, M. M. S., Haslett, M. I. C., Freitas, L. J. A. D., Gomes, N. T. N., Silva, D. C. C. D., Percio, J., \& Alves, A. J. S. (2020). Descrição dos casos hospitalizados pela COVID-19 em profissionais de saúde nas primeiras nove semanas da pandemia, Brasil, 2020. Epidemiologia e Serviços de Saúde, 29, e2020277. https://doi.org/10.1590/S1679-49742020000500011

Gallasch, C. H., da Cunha, M. L., de Souza Pereira, L. A., \& Silva-Junior, J. S. (2020). Prevenção relacionada à exposição ocupacional do profissional de saúde no cenário de COVID-19. Revista Enfermagem UERJ, 28, 49596. https://doi.org/10.12957/reuerj.2020.49596

Galvão, T. F., Pansani, T. D. S. A., \& Harrad, D. (2015). Principais itens para relatar Revisões sistemáticas e Meta-análises: A recomendação PRISMA. Epidemiologia e Serviços de Saúde, 24, 335-342. http://dx.doi.org/10.5123/S1679-49742015000200017

Gomez, C. M., Vasconcellos, L. C. F. D., \& Machado, J. M. H. (2018). Saúde do trabalhador: aspectos históricos, avanços e desafios no Sistema Único de Saúde. Ciência \& Saúde Coletiva, 23, 1963-1970. https://doi.org/10.1590/1413-81232018236.04922018

Hopia, H., et al. (2016). Reviewing the methodology of an integrative review. Scand J Caring Sci. 30, 662-669. https://doi.org/10.1111/scs.12327

Lima, D. S., Leite Filho, J. A. D., Gurgel, M. V. S. A., de Aguiar Neto, A. F., da Costa, E. D. F. M., Maia Filho, F. X. F., \& Junior, M. A. F. R. (2020). Recomendações para cirurgia de emergência durante a pandemia do COVID-19. Journal of Health \& Biological Sciences, 8(1), 1-3. http://dx.doi.org/10.12662/2317-3076jhbs.v8i1.3176.p1-3.2020

Melnyk, B. M., et al. (2010). Evidence-based practice: step by step: the seven steps of evidence-based practice. AJN The American Journal of Nursing. 110(1), 51-53. https://doi.org/10.1097/01.naj.0000366056.06605.d2

Mendes, K. D. S., et al. (2008). Revisão integrativa: método de pesquisa para a incorporação de evidências na saúde e na enfermagem. Texto Contexto Enferm. 17(4), 758-764. https://doi.org/10.1590/S0104-07072008000400018

Miranda, F. M. D. A., de Lima Santana, L., Pizzolato, A. C., \& Sarquis, L. M. M. (2020). Condições de trabalho e o impacto na saúde dos profissionais de enfermagem frente a Covid-19. Cogitare enfermagem, 25. http://dx.doi.org/10.5380/ce.v25i0.72702

Moran, C. (2017). Introducing emotional freedom techniques. Routledge.

Oliveira, E. C. D. S., Silva, F. P. D., Pereira, E. B. F., \& Oliveira, R. C. D. (2020). Ações da comissão de controle de infecção hospitalar frente ao novo coronavírus. Rev. baiana enferm, e37259-e37259. https://doi.org/10.18471/rbe.v34.37259

Pires, M. (2020). As principais reações à crise. Observatório de Política Fiscal. Instituto Brasileiro de Economia. https://observatorio-politicafiscal.ibre.fgv.br/sites/observatorio-politica-fiscal.ibre.fgv.br/files/u52/as_principais_reacoes_a_crise_0.pdf

Sousa, I. M. C. D., \& Tesser, C. D. (2017). Medicina Tradicional e Complementar no Brasil: inserção no Sistema Único de Saúde e integração com a atenção primária. Cadernos de Saúde Pública, 33. https://doi.org/10.1590/0102-311X00150215

Shawahna, R. (2021). Knowledge, attitude, and use of protective measures against COVID-19 among nurses: a questionnaire-based multicenter cross-sectional study. BMC nursing, 20(1), 1-13. https://doi.org/10.1186/s12912-021-00689-X

Tang, R., Ranmuthugala, G., \& Cunningham, F. (2014). Surgical safety checklists: a review. ANZ journal of surgery, 84(3), 148-154. https://doi.org/10.1111/ans. 12168 
Research, Society and Development, v. 10, n. 12, e164101219882, 2021

(CC BY 4.0) | ISSN 2525-3409 | DOI: http://dx.doi.org/10.33448/rsd-v10i12.19882

Tobase, L., Cardoso, S. H., Rodrigues, R. T. F., \& Peres, H. H. C. (2021). Empathic listening: welcoming strategy for nursing Professional in coping with with the coronavirus pandemic. Revista brasileira de enfermagem, 74. https://doi.org/10.1590/0034-7167-2020-0721

Trigueiro, R. L., Araújo, A. L. D., Moreira, T. M. M., \& Florêncio, R. S. (2020). COVID-19 pandemic: report on the use of auriculotherapy to optimize emergency workers' health. Revista brasileira de enfermagem, 73. https://doi.org/10.1590/0034-7167-2020-0507

Whittemore, R., \& Knafl, K. (2005). The integrative review: updated methodology. J Adv Nurs. 52(5), 546-553. https://doi.org/10.1111/j.13652648.2005.03621.x

World Health Organization. (2020). Coronavirus disease (COVID-19) outbreak: rights, roles and responsibilities of health workers, including key considerations for occupational safety and health: interim guidance, 19 March 2020 (No. WHO/2019-nCov/HCW_advice/2020.2). https://apps.who.int/iris/bitstream/handle/10665/331510/WHO-2019-nCov-HCWadvice-2020.2-eng.pdf 